\title{
Morphological and functional positive effect with the association of hyperbaric oxygen therapy and corticoid against acute acoustic trauma
}

\author{
Gleice Cristina Colombari ${ }^{1}$, Adriana de Andrade Batista Murashima ${ }^{1}$, Omar Feres ${ }^{2}$, \\ Miguel Angelo Hyppolito ${ }^{1}$ \\ ${ }^{1}$ Division of Otorhinolaryngology, Department of Ophthalmology, Otorhinolaryngology and Head and Neck Surgery, Faculty of Medicine of \\ Ribeirão Preto, University of São Paulo, Ribeirão Preto, São Paulo, Brazil \\ ${ }^{2}$ Division of Surgery, Department of Anatomy and Surgery, Faculty of Medicine of Ribeirão Preto, University of São Paulo, Ribeirão Preto, \\ São Paulo, Brazil
}

\section{Emailaddress:}

gcolombari@gmail.com (G. C. Colombari),gleice.colombari@usp.br (G. C. Colombari), adrimurashima@gmail.com (A. D. A. B. Murashima), mahyppo@fmrp.usp.br (M. A. Hyppolito), feresomar@netsite.com.br (O. Feres)

\section{To cite this article:}

Gleice Cristina Colombari, Adriana de Andrade Batista Murashima, Omar Feres, Miguel Angelo Hyppolito. Morphological and Functional Positive Effect with the Association of Hyperbaric Oxygen Therapy and Corticoid Against Acute Acoustic Trauma. American Journal of Biomedical and Life Sciences. Special Issue: Mechanisms of Protection Against Oxidative Stress. Vol. 2, No. 6-1, 2014, pp. 13-18. doi: $10.11648 /$ j.ajbls.s.2014020601.13

\begin{abstract}
Studies describe the use of corticosteroids as the best alternative to treat acoustic trauma, but recent studies point to their combination with hyperbaric oxygen therapy (HBOT) as the major benefit in lesions by noise. This study aimed to evaluate the association between HBOT and corticoid therapy (CT). Female guinea pigs were exposed to a white noise on 4 $\mathrm{kHz}$ at $110 \mathrm{~dB}$ SPL during 72 hours and divided into three treatment groups: HBOT, CT and the combination of both therapies (HBOCT). The treatment lasted five days, being a therapy session per day. All animals were exposed to Distortion Product Otoacoustic Emissions and Brainstem Auditory Evoked Potentials in three moments: before and after exposure to the noise and after the treatment. All cochleae were examined by scanning electron microscopy. Although not statistically significant, the anatomical and functional findings concluded that the association HBOCT played a better otoprotective and therapeutic effect compared to those same therapies alone.
\end{abstract}

Keywords: Acoustic Trauma, Otoprotection, Hyperbaric Oxygen Therapy

\section{Introduction}

The hearing organ exposure to acoustic superstimulation by an intense impulse sound, may cause a sudden sensorineural hearing loss and occur due to altered cochlear oxygenation, decreased cochlear blood flow, damage to cell membranes, metabolic effects of free radicals, altered neurotransmission and apoptosis [1-6].

For many years, several treatments of acoustic trauma have been used to reduce inflammation, to enhance cochlear oxygenation and to remove the metabolites that occur during intense function of the outer hair cells (OHCs) $[7,8]$.

According to studies performed in the last decade, the treatment most often used in acoustic trauma with greatest therapeutic benefits is the combination of hyperbaric oxygen therapy (HBOT) with corticoid therapy (CT) [2,7,8]. The effectiveness of this combination therapy has been demonstrated in hearing losses of mild and moderate severity $[2,7,8]$.

Fakhry et al. [8] proposed that the hearing improvement obtained with the combination of HBOT with $\mathrm{CT}$ for acoustic trauma appeared not to have been proportional to the induced hearing loss but rather to have exerted a constant and limited effect when compared to previous studies conducted by d'Aldin et al. [2] and Lamm and Arnold [7]. They justify this argument due to the similar magnitude of hearing loss reduction (about $10-15 \mathrm{~dB}$ ) as in previous studies, although the induced hearing loss was considerably greater (about 40 $\mathrm{dB}$ instead of $20-25 \mathrm{~dB}$ ).

Given the lack of studies and the need to correlate the 
functional and morphological findings, the objective of the present study was to evaluate the combination of HBOT and CT used in acoustic trauma by the analysis of the functional and morphological findings.

\section{Material and Methods}

\subsection{Animals}

Eighteen female guinea pigs were used, cycled in diestrus (Cavia porcellus) and weighing 350 to $500 \mathrm{~g}$. All procedures were previously approved by the Ethics Committee for Animal Experiments (CETEA) of the Faculty of Medicine of RibeirãoPreto, University of São Paulo (FMRP-USP) ( ${ }^{\circ}$ 072/2009) according to the recommendations of the Brazilian College of Animal Experimentation (COBEA) for care with experimental animals.

The animals were selected according to the Preyer reflex [9]. After 24 hours of hearing rest, the animals were reevaluated by otoscopy. Animals with signs of external otitis or acute otitis media, earwax of difficult removal, inflammatory changes of the external auditory canal or a very narrow auditory canal impairing a proper accommodation of the otoacoustic emission probe in at least one of the ears were excluded from the study.

\subsection{Brainstem Auditory Evoked Potentials and Distortion Products Otoacoustic Emission}

Before the exams, all animals were anesthetized with ketamine hydrochloride, an anesthetic drug with no effect on auditory function.

Surface electrodes were used for the recording of brainstem auditory evoked potentials (BAEP), arranged as follows: a positive electrode on the skull vertex, one negative electrode on the posterior portion of each ear pavilion, and a reference (ground) electrode on the forehead (between the eyes). For better conductivity of the electric signal, the regions of the vertex and between the ocular orbits were shaved and the electrodes were positioned with the interposition of electrolytic paste. Acquisition was ipsilateral, with a time of analysis of $12 \mathrm{~ms}$ and a sample of 1000 stimulus passes, with replication. A high-pass $50 \mathrm{~Hz}$ filter and a low pass $3 \mathrm{kHz}$ filter were used. The stimulus used was a 27.7/s click emitted by Ear Tone $3 \mathrm{~A}$ insertion phones in alternate polarity. The electrophysiological hearing threshold was determined by the lowest intensity at which wave II was identified.

For the distortion products otoacoustic emission (DPOAE) exam, two simultaneous pure tones (F1 and F2) were used, with $\mathrm{F} 1<\mathrm{F} 2$, at frequencies of $1,1.5,2,3$ and $4 \mathrm{kHz}$ with resolution of 3 points per octave, at the intensity of $70 \mathrm{~dB}$ SPL. The analysis of the DPOAE results were based on the signal-to-noise ratio (SNR) values that indicate the difference between the otoacoustic emissions (OAE) response and the noise level at a particular frequency.

The equipment used for the exams was the Smart EP and DPOEA of Intelligent Hearing Systems (Miami, FL, USA).

\subsection{Acoustic Trauma}

To induce the acoustic trauma, the guinea pigs were bilaterally exposed to the same stimulus and methods described by Colombari, et al. [10], a white noise on $4 \mathrm{kHz}$ at $110 \mathrm{~dB}$ SPL during 72 hours.

\subsection{Hyperbaric Oxygen Therapy and Corticoid Therapy}

An experimental hyperbaric chamber directly pressurized with oxygen $\left(\mathrm{O}_{2}\right)$ was used for the HBOT sessions. Three animals at a time were submitted to daily therapeutic hyperbaric oxygen (HBO) sessions for five consecutive days. The sessions consisted of 15 minutes of compression up to 2.3 ATA, followed by one hour at this level and then by 15 minutes of decompression.

Corticoid therapy (CT) consisted of systemic administration of dexamethasone $(10 \mathrm{mg} / \mathrm{kg} / \mathrm{day})$ for five consecutive days.

\subsection{Execution of the Experiment}

Eighteen animals were randomly divided into three groups of six. Three study situations were proposed according to the therapeutic method used: CT, HBOT and the combination of both therapies, HBOCT.

Three times of audiological evaluation were used for each situation (pre-noise, post-noise and post-treatment). The prenoise time referred to the exams (BAEP and DPOAE) performed before exposure to noise, the post-noise time referred to the exams performed after the acoustic trauma, and the post-treatment time referred to the exams performed after the therapeutic intervention.

\subsection{Scanning Electron Microscopy (SEM)}

After euthanasia of the animals at the scheduled time points, all cochleae were prepared as described by Colombari, et al. [10], analyzed and photographed with a JEOL scanning microscope JSM 5200.

\subsection{Statistical Analysis}

A fully randomized experimental design was adopted, with each ear being defined as an experimental unit. Analysis of variance followed by the Tukey test $(p<0.05)$ were used to compare the dependent variables, electrophysiological hearing threshold (BAEP) and SNR (DPOAE), at the different times of evaluation (within group) and the amplitudes of these variables between the different situations proposed (between groups).

Analysis of variance were performed using the SAS ${ }^{\circ}$ software for Windows version 9.2 (Statistical Analysis System - SAS Institute, 2011).

\section{Results}

There were highly significant differences $(p \leq 0.01)$ in the mean values of the two variables, i.e., SNR and electrophysiological auditory threshold, between the pre- 
noise evaluation ("a" by the Tukey test) and the post-noise evaluation ("b"). The SNR's mean showed a significant reduction of 17.20 in the pre-noise value in the post-noise situation and the mean value of the electrophysiological hearing threshold increased significantly by $46.27 \mathrm{~dB}$ after exposure to noise ("a").

In all the study situations proposed, i.e., CT, HBOT and HBOCT, there was a highly significant difference $(p \leq 0.01)$ in the analysis of variance for the effect between times, within the situations for the SNR of the DPOAE of all the frequencies studied. The Tukey test revealed that the prenoise time differed significantly from the remaining times of evaluation, characterizing two distinct groups, the first defined by the pre-noise time ("a") and the second by the post-noise and post-treatment times ("b"). Although the postnoise and post-treatment times did not differ significantly, an improvement was observed in the SNR after treatment with all the therapeutic methods used.

Regarding the electrophysiological hearing threshold variable, regardless of the situation, the post-noise time was characterized as the time of the highest degree of hearing loss, as demonstrated by the significantly higher mean values differing from the remaining ones ("a"). The post-treatment time was characterized by a significant hearing loss of lower degree in relation to the post-noise time ("b"), but not significant compared to the pre-noise time ("c"). The group submitted to CT showed a significant mean reduction of the post-treatment electrophysiological hearing threshold of 20.4 $\mathrm{dB}$, with a difference of $25.4 \mathrm{~dB}$ from the pre-noise situation; The group submitted to HBOT showed a significant mean reduction of the post-treatment electrophysiological hearing threshold of $15.4 \mathrm{~dB}$, differing by $25.9 \mathrm{~dB}$ from the pre-noise time, and the group submitted to HBOCT, with a mean increase in the electrophysiological hearing threshold of 43.3 $\mathrm{dB}$, showed a mean reduction of the electrophysiological hearing threshold of $22.9 \mathrm{~dB}$, with a difference of $20.4 \mathrm{~dB}$ from the initial situation.
Analyses of variance for the amplitudes of the variables studied between the post-treatment and pre-noise and the post-treatment and post-noise times were performed in order to compare the different situations. For the amplitudes of the variable SNR of the DPOAE, the F test was nonsignificant in all situations, with no difference in mean amplitudes after treatment both regarding pre-noise and post-noise in the various situations. The $\mathrm{F}$ test was nonsignificant for the amplitudes of the electrophysiological hearing threshold of post-treatment both for pre-noise and post-noise in all situations, i.e., there was no difference in these amplitudes between the three situations (Table 1).

Analysis by SEM showed that the cochlear specimens of the animals submitted only to HBOT (Fig. 1) presented ciliary distortions and loss of isolated stereocilia only from the first row of OHCs of the middle third to the initial third of the second turn. Greater damage extending to the three $\mathrm{OHCs}$ rows and a greater loss of stereocilia occurred from the middle third of the second turn to the initial third of the third turn. The middle third of the third turn was characterized more by ciliary distortions than by stereocilia losses, and a progressive improvement of damage was noted from the final third to the apical turn, approaching the habitual anatomical pattern.

The cochlear specimens of the animals submitted only to CT (Fig. 2) showed isolated ciliary distortions and loss of stereocilia concentrated in the first OHCs row in the middle and final thirds of the basal turn. The distortion and ciliary losses were more marked in the second turn than in the basal turn and in the three OHCs rows. In the transition from the second to the third turn a much more defined pattern of injury was observed, with a greater quantity of ciliary loss and with more extensive ciliary distortions that were maintained up to the middle third. A progressive pattern of improvement of the injuries was observed in the direction of the apical third, approaching the habitual anatomical pattern.

Table 1. Factors of variation (FV), degrees of freedom (DF) and mean squares (MS) with significance according to the F test, coefficient of variation (CV\%), experimental accuracy $(A c)$ and general mean (GM) obtained by individual analyses of variance for the amplitude of the electrophysiological hearing threshold (in $\mathrm{dB}$ ), of post-treatment with pre-noise and with post-noise for the situations (CT, HBOT and HBOCT) and their respective mean amplitudes

\begin{tabular}{|c|c|c|c|c|c|c|}
\hline \multirow{2}{*}{ FV } & \multicolumn{3}{|c|}{ Amplitude between post-treatment and pre-noise } & \multicolumn{3}{|c|}{ Amplitude between post-treatment and post-noise } \\
\hline & DF & MS & & DF & MS & \\
\hline Treatments & 2 & 72.7 & ns & 2 & 116.7 & ns \\
\hline Residue & 51 & 202.1 & & 51 & 185.0 & \\
\hline $\mathrm{CV} \%$ & 89.27 & & & 104.20 & & \\
\hline Ac & 0.9307 & & & 0.9587 & & \\
\hline GM & 15.93 & & & 13.06 & & \\
\hline $\mathrm{CT}^{[1]}$ & 16.94 & & a & 13.61 & & $\mathrm{a}$ \\
\hline $\mathrm{HBOT}^{[2]}$ & 17.22 & & a & 10.28 & & a \\
\hline $\mathrm{HBOCT}^{[3]}$ & 13.61 & & $\mathrm{a}$ & 15.28 & & a \\
\hline
\end{tabular}

$*, * *$ and ${ }^{\mathrm{ns}}: \mathrm{F}$ test significant at $5 \%, 1 \%$ and nonsignificant, respectively;

${ }^{\mathrm{a}}$ : Tukey test - means followed by the same letters did not differ at the $5 \%$ level; ${ }^{[1]} \mathrm{CT}=$ corticoid therapy ${ }^{[2]} \mathrm{HBOT}=$ hyperbaric oxygen therapy; ${ }^{[3]} \mathrm{HBOCT}$

$=$ combination of HBOT and $\mathrm{CT}$ 

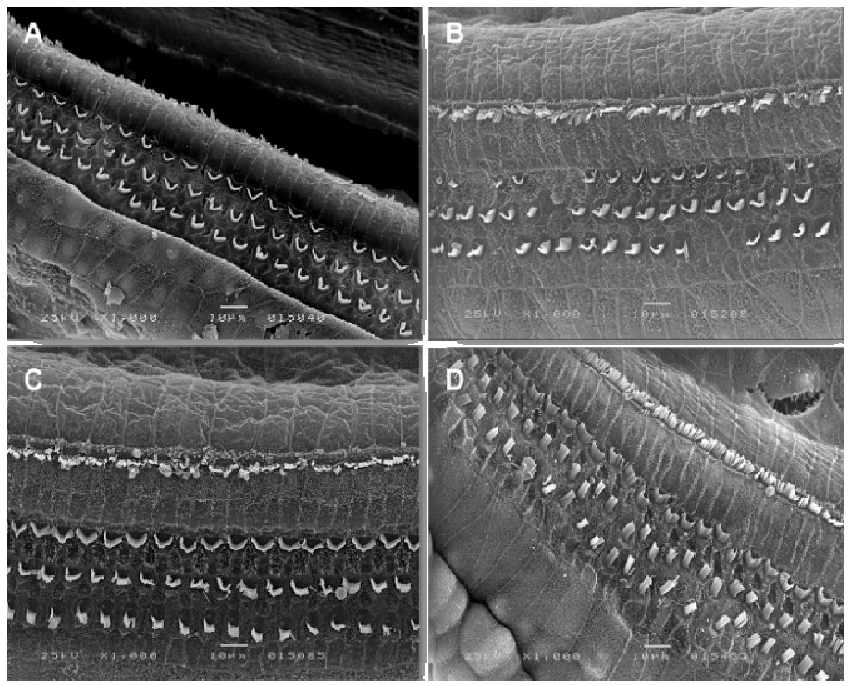

Figure 1. Scanning electron microscopy photographs, with magnification of 1000 times, from cochlear specimens of guinea pigs treated during five days with hyperbaric oxygen therapy (1 session/day) after exposure to a $4 \mathrm{kHz}$ octave band noise with a $110 \mathrm{~dB} S P L$ intensity for 72 hours. Letter A refers to middle third of the basal turn; $B$, to middle third of the second turn; $C$, to middle third of the third turn and $D$, to middle third of the apical.
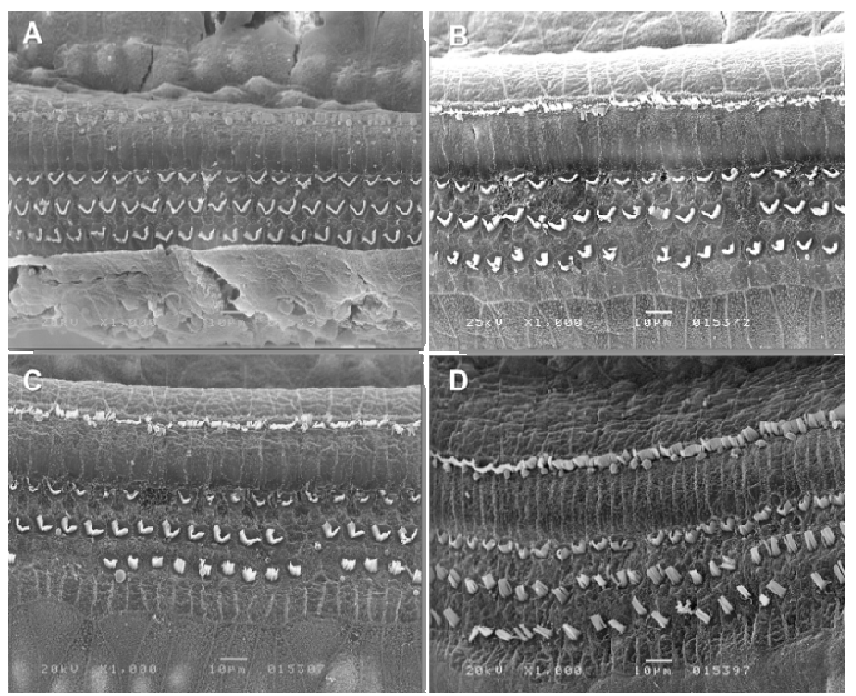

Figure 2. Scanning electron microscopy photographs, with magnification of 1000 times, from cochlear specimens of guinea pigs treated during five days with dexamethasone $(10 \mathrm{mg} / \mathrm{kg} /$ day) after exposure to a $4 \mathrm{kHz}$ octave band noise with a $110 d B$ SPL intensity for 72 hours. Letter A refers to middle third of the basal turn; $B$, to middle third of the second turn; $C$, to middle third of the third turn and $D$, to middle third of the apical.

The cochlear specimens treated with HBOCT (Fig. 3) showed ciliary distortions and highly isolated stereocilia loss, concentrated in the first $\mathrm{OHCs}$ row, in the middle and final thirds of the basal turn and in the initial third of the second turn. Ciliary distortions in the three OHCs rows were observed in the middle and final thirds of the second turn, but were more evident in the first turn. Continuity of the pattern of damage was observed in the initial third of the third turn, although the first row was less compromised. Progressive improvement of the injury was noted from the middle to the apical third, approaching the habitual anatomical patter.

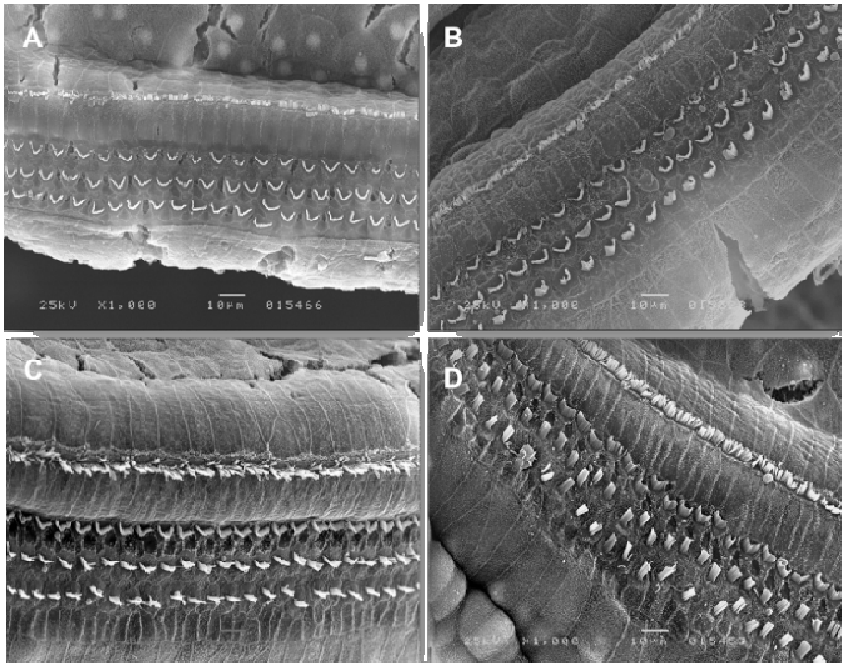

Figure 3. Scanning electron microscopy photographs, with magnification of 1000 times, from cochlear specimens of guinea pigs treated during five days with hyperbaric oxygen therapy (1 session/day) combined with dexamethasone $(10 \mathrm{mg} / \mathrm{kg} /$ day) after exposure to a $4 \mathrm{kHz}$ octave band noise with a $110 \mathrm{~dB} S P L$ intensity for 72 hours. Letter A refers to middle third of the basal turn; $B$, to middle third of the second turn; $C$, to middle third of the third turn and $D$, to middle third of the apical.

\section{Discussion}

Acute acoustic trauma is a sudden onset of sensorineural hearing impairment due to exposure of the hearing organ to acoustic overstimulation, typically by intense impulse noise [11]. Acoustic trauma results in structural changes such as rupture of the cell membranes, reduction of cochlear blood flow and destruction of cochlear hair cellsand/or dendrites of primary auditory neurons [1-5].

Changes in oxygenation and decreased cochlear blood flow appear to occur first, inducing an accumulation of free radicals in the cochlea that triggers processes of dysfunction and cell death [12-15].

The various treatments proposed for acoustic trauma have focused on the reduction of inflammation and the promotion of cochlear oxygenation. According to Lamm and Arnold [16], the administration of anti-inflammatory drugs in the presence of noise-induced cochlear changes isbased on the fact that inflammatory tissue changes are not only provoked by bacteria, viruses or other immunopathological processes, but also by cell damage, hypoxia and tissue ischemia.

Corticoids are the most potent anti-inflammatory drugs and their use has been affective for the treatment of acoustic trauma [2,16-19].

In the present study, the group submitted to CT, although not differing significantly in terms of the SNR of the DPOAE, showed a significant mean reduction of the electrophysiological hearing threshold of $20.4 \mathrm{~dB}$, with a difference of $25.4 \mathrm{~dB}$ compared to the pre-noise situation.

Thus, the present findings confirm the effectiveness of CT for acoustic trauma, in agreement with previous studies [2, 16-19].

Different corticoid doses for the treatment of acoustic 
trauma have been reported in the literature, ranging from 2 to $200 \mathrm{mg} / \mathrm{kg}$ [2, 7, 8, 19]. Although d'Aldin et al. [2] concluded that only the corticoid dose of $20 \mathrm{mg} / \mathrm{kg}$ induced a significant improvement of the thresholds, the dose of 10 $\mathrm{mg} / \mathrm{kg} /$ day chosen for the present investigation was based on the study by Fakhry et al. [8], who demonstrated a reduction of hearing thresholds with the same dose.

It should be pointed out that d'Aldin et al. [2] studied doses of 2, 20, 40, 100 and $200 \mathrm{mg} / \mathrm{kg}$, a fact that does not rule out the possibility of effectiveness of the dose of 10 $\mathrm{mg} / \mathrm{kg}$. However, we should still question the possibility of a more expressive improvement with higher doses than those used in the present study.

HBOT increases the oxygen levels in a more effective manner [20], also favoring repair of the microcirculation and permitting its use in the treatment of various diseases, including sensorineural hearing loss [21]. As previously reported in the literature HBOT treatment for acoustic trauma provides an appropriate oxygen supply, avoiding cochlear hypoxia that starts the oxidative stress responsible for apoptosis cascade, that trigger hair cells death [3,11].

In the group submitted only to HBOT, the mean SNR of the DPOAE also did not differ significantly between the times of evaluation post-noise and post-treatment. However, the electrophysiological hearing threshold was significantly reduced by $15.4 \mathrm{~dB}$ after treatment, differing by $25.9 \mathrm{~dB}$ from the pre-noise time. These findings support the existence of the therapeutic effect of HBO on acoustic trauma [10].

Studies have pointed out that HBOT potentiates CT, with this combination having the best therapeutic effect among the treatments used for noise-induced hearing loss $[2,7,8]$.

The effectiveness of this combined therapy was demonstrated by mild losses of 20 to $25 \mathrm{~dB}[2,7]$ and by moderate hearing losses of about $40 \mathrm{~dB}$ [8]. This reduction of hearing thresholds was similar to that demonstrated by Fakhry et al. [8], Lamm and Arnold [7] and d'Aldin et al. [2].

Considering the mean increase in the post-noise electrophysiological hearing threshold of $45.6 \mathrm{~dB}$, the proposed situation of the therapeutic combination, HBOCT, led to a mean reduction of $33.9 \mathrm{~dB}$ of the electrophysiological hearing threshold, differing $20.4 \mathrm{~dB}$ from the value of the initial situation. This reduction was greater than that of 10 to $15 \mathrm{~dB}$ reported by Fakhry et al. [8] for hearing losses of $40 \mathrm{~dB}$. Present results contradict the constant and limited effect of the therapeutic combination of HBOCT and corticoid predicted by the literature. This argument is also reinforced by the treatment time used in the present study compared to the study of Fakhry et al. [8], of 5 and 10 days, respectively.

Post-noise-treatment amplitudes had showed lower amplitude between post-treatment and pre-noise and greater amplitude between post-treatment and post-noise in HBOCT and CT group, with greater amplitude obtained with HBOT (HBOCT $<\mathrm{CT}<\mathrm{HBOT}$ ). Regarding the post-treatment and post-noise amplitude, the highest values were obtained with HBOCT (HBOCT $>$ CT $>$ HBOT), showing a better therapeutic performance observed for the HBOCT.

Our experimental findings by functional and anatomically assessment by SEM reinforce that the combination of HBOT and CT led to a greater cochlear recovery after acoustic trauma compared to each treatment alone.

SEM revealed that, regardless of the therapeutic method applied, the initial third of the basal turn (BT) did not show any changes and the middle third of the same turn showed only some ciliary distortions and/or loss of stereocilia. In contrast, the final BT turn of the groups submitted to HBOT differed from the remaining groups, with distortions and/or ciliary losses of greater amplitude.

The group submitted to HBOCT showed a less altered initial third of the second turn (T2) compared to the groups submitted to each therapy alone. The middle and final thirds of this turn showed a closely similar profile in the $\mathrm{CT}$ and HBOCT groups, but showed a pattern closer to the habitual one compared to the group treated with HBOT.

The third turn (T3) showed a much better pattern throughout its extension in the HBOCT group compared to the HBOT and CT groups.

Exposure to intense noise may provoke damage such as derangement, collapse, losses, fusions or elongation of the cilia of auditory sensory cells. It has also been reported that hair cells may also show breakage of part or all of their sensory cilia [22].

Zuo et al. [23] observed loss and derangement of OHCs stereocilia as the morphological injuries of cochleae exposed to noise.

The spectrum of anatomical changes in the stereocilia of sensory cells (OHCs) detected in the specimens analyzed agreed with data reported in the literature.

Saunders et al. [22] showed that mechanically induced injuries usually recover rapidly, whereas those induced by metabolic exhaustion show a more gradual recovery.

Stengs et al. [24] suggested a self-defense mechanism regarding the spontaneous recovery of injured $\mathrm{OHC}$, but Cardinaal et al. [25] stated that these same findings do not permit a conclusion about the question of the occurrence of recovery of damaged cells or the formation of new cells. Synaptic repair may occur 5 days after noise injury, but the damaged cells persist $[2,10]$.

Guinea pigs exposed to noise did not recover after five days of rest and, when compared to the immediate post-noise situation, showed even greater injury due to the metabolic damage present even after the cessation of the damaging stimulus [10].

Lamm and Arnold [7] and D'Aldin et al. [2] concluded that, despite an incomplete recovery, intervention by HBOT plus CT resulted in a significant morphological recovery.

It can be seen that, even though the $\mathrm{CT}$, HBOT and HBOCT situations did not differ significantly in the evaluation of functional hearing, the tendency to a better therapeutic efficacy in the HBOCT situation could also be visualized by SEM in view of the greater anatomical preservation, with the occurrence of greater recovery and/or greater otoprotection against the metabolic injuries. 


\section{Conclusion}

Therapeutic combination of HBOT with $\mathrm{CT}$ was more effective to acute acoustic trauma than each treatment HBOT or CT, applied alone.

\section{References}

[1] E. Chan, A. Suneson, and M. Ulfendahl, "Acoustic trauma causes reversible stiffness changes in auditory sensory cells,"Neuroscience. Oxford, vol. 83, n. 3, pp. 961-968, April 1998.

[2] C. D'Aldin, L. Cherny, F. Devriére, and A. Dancer, "Treatment of Acoustic Trauma," Ann. N. Y. Acad. Sci. New York, vol. 884, n. 28, pp. 328-344, November 1999.

[3] J. Kuokkanen, A. A. Aarnisalo, and J. Ylikoski, "Efficiency of hyperbaric oxygen therapy in experimental acute acoustic trauma from firearms," Acta. Otolaryngol. Suppl. Oslo, vol. 120, n. 1, pp.132-134, January 2000.

[4] T. Endo, T. Nakagawa, F. Iguchi, T. Kita, T. Okano, S. H. Sha, J. Schacht, A. Shiga, T. S. Kim, and J. Ito, "Elevation of superoxide dismutase increases acoustic trauma from noise exposure,". Free Radical Biology \& Medicine Tarrytown, vol. 38, n. 4, pp. 492-498, February 2005.

[5] B. O. Çakir, I. Ergan, S. Civelek, S. Körpinar, A. S. Toklu, O. Gedik, G. Isik, I. Sayin, and S. Turgut, "Negative Effect of immediate hyperbaric oxygen therapy in acute trauma," Otol. Neurotol. Hagerstown, vol. 27, n. 4, pp. 478-483, June 2006.

[6] D. Henderson, E. C. Bielefeld, K. C. Harris, and B. H. Hu "The role of oxidative stress in noise-induced hearing loss," Ear Hear. Baltimore, vol. 27, n. 1, pp. 1-19, February 2006

[7] K. Lamm, and W. Arnold, "Successful Treatment of NoiseInduced Cochlear Ischemia, Hypoxia, and Hearing Loss," Ann. N.Y. Acad. Sci. New York, vol. 884, n. 28, pp. 233-248, November 1999.

[8] N. Fakhry, J. C. Rostain, and Y. Cazals, "Hyperbaric oxygenation with corticoid in experimental acoustic trauma," Hear. Res. Amsterdam, vol. 230, n. 4, pp. 88-92, August 2007.

[9] J. Jero, D. E. Coling, and A. K. Lalwani, "The use of Preyer's reflex in evaluation of hearing in mice," ActaOtolaryngol. Oslo, vol. 121, n. 5, pp. 585-589, July 2001.

[10] G. C. Colombari, M. Rossato, O. Feres, and M. A. Hyppolito, "Effects of hyperbaric oxygen treatment on auditory hair cells after acute noise damage," Eur. Arch. Otorhinolaryngol. Heidelberg, vol. 268, n. 1, pp. 49-56, January 2011.

[11] J. Ylikoski, R. Mrena, A. Mäkitie, J. Kuokkanen, U. Pirvola, and S. Savolainen, "Hyperbaric oxygen therapy seems to enhance recovery from acute acoustic trauma," ActaOtoLaryng. Stockholm, vol. 128, n.10, pp. 1110-1115, April 2008.

[12] P. R., Thorne, and A. L. Nuttall, "Alterations in oxygenation of cochlear endolymph during loud sound exposure," ActaOtolaryngol. Stockholm, vol. 107, n. 1-2, pp. $71-79$, January - February 1989.
[13] H. Yamane, Y. Nakai, M. Takayama, H. Iguchi, T. Nakagawa, and A. Kojima, "Appearance of free radicals in the guinea pig inner ear after noise-induced acoustic trauma," Eur Arch Otorhinolaryngol. Heidelberg, vol. 252, n .8, pp. 504-508, August 1995.

[14] K. Lamm, and W. Arnold, "Noise-induced cochlear hypoxia is intensity dependent, correlates with hearing loss and precedes reduction of cochlear blood flow," Audiol. Neurootol. vol. 1, n. 3, pp.148-160, Basel, May-June 1996.

[15] D. Yamashita, H. Y. Jiang, C. G. Le Prell, J. Schacht, and J. M. Miller, "Post-exposure treatment attenuates noise-induced hearing loss," Neuroscience New York, vol. 134, n. 2, pp. 633 -642, January 2005.

[16] K. Lamm, and W. Arnold, "The effect of prednisolone and non-steroidal anti-inflammatory agents on the normal and noise-damaged guinea pig inner ear," Hear. Res. Amsterdam, vol. 115, n.1-2, pp. 149-161, January 1998.

[17] K. Takahashi, J. Kusakari, S. Kimura, T. Wada, and A. Hara, "The effect of methylprednisolone on acoustic trauma," ActaOtolaryngol. Stockholm, vol. 116, n. 2, pp. 209-212, March 1996.

[18] Y. Tahera, I. Meltser, P. Johansson, Z. Bian, P. Stierna, A. C. Hansson, and B. Canlon, "NF-kappaB mediated glucocorticoid response in the inner ear after acoustic trauma," J. Neurosci. Res. New York, vol. 83, n. 6, pp. 1066-1076, May 2006.

[19] H. H. Arslan, B. Satar, M. A. Serdar, M. Ozler and E. Yilmaz, "Effects of hyperbaric oxygen and dexamethasone on proinflammatory cytokines of rat cochlea in noise-induced hearing loss," Otolology\&Neurotology. Hagerstown, vol. 33, n.9, pp.1672-1678, December 2012.

[20] P. M. Tibbles, and J. S. Edelsberg, "Hyperbaric-oxygen therapy,” N. Engl. J. Med. Boston, vol. 334, n. 25, pp. 1642 1648, June. 1996.

[21] D. J. Bakker, "Hyperbaric oxygen therapy: Past, present and future indications," Adv. Exp. Med. Biol. New York, vol. 317, pp. 95-105, 1992.

[22] J. C. Saunders, S. P. Dear, and M. E. Scheneider, "The anatomical consequences of acoustic injury: A review and tutorial," J. Acoustic. Soc. Am Melville, vol. 78, n. 3, pp. 833860, September 1985.

[23] H. Zuo, B. Cui, X. She., and M. Wu. "Changes in Guinea pig cochlear hair cells after sound conditioning and noise exposure," J. Occup. Health.Tokyo, vol. 50, n. 5, pp. $373-$ 379, July 2008.

[24] C. H. M. Stengs, S. F. L. Klis, E. H. Huizing, and G. F. Smoorenburg, "Cisplatin-induced ototoxicity; electrophysiological evidence of spontaneous recovery in the albino guinea pig," Hear. Res., Amesterdam, vol.111, n.1-2, pp.103-113, September 1997.

[25] R. M. Cardinaal, J. C. M. J. Groot, E. H. Huizing, J. E., and G. F. Smoorenburg, "Cisplatin-induced ototoxicity: morphological evidence of spontaneous outer hair cell recovery in albino guinea pigs?," Hear. Res. Amsterdam, vol. 144, n. 1-2, pp.147-156, June 2000. 